\title{
Polar accumulation of the metabolic sensory histidine kinases DcuS and CitA in Escherichia coli
}

\begin{abstract}
Correspondence
Wolfgang Erker

erker@uni-mainz.de
\end{abstract}

Received 18 March 2008

Revised 22 April 2008

Accepted 8 May 2008

\section{Patrick Scheu, ${ }^{1} \dagger$ Sven Sdorra,${ }^{2} \dagger$ Yun-Feng Liao, ${ }^{2}$ Maria Wegner, ${ }^{2}$ Thomas Basché, ${ }^{2}$ Gottfried Unden ${ }^{1}$ and Wolfgang Erker ${ }^{2}$}

\author{
${ }^{1}$ Institute of Microbiology and Wine Research, Johannes Gutenberg University, Mainz, Becherweg \\ 15, 55099 Mainz, Germany \\ ${ }^{2}$ Institute of Physical Chemistry, Johannes Gutenberg University, Mainz, Welderweg 11, 55099 \\ Mainz, Germany
}

\begin{abstract}
Signal transduction in prokaryotes is frequently accomplished by two-component regulatory systems in which a histidine protein kinase is the sensory component. Many of these sensory kinases control metabolic processes that do not show an obvious requirement for inhomogeneous distribution within bacterial cells. Here, the sensory kinases DcuS and CitA, two histidine kinases of Escherichia coli, were investigated. Both are membrane-integral and involved in the regulation of carboxylate metabolism. The two-component sensors were fused with yellow fluorescent protein (YFP) and live images of immobilized cells were obtained by confocal laser fluorescence microscopy. The fluorescence of the fusion proteins was concentrated at the poles of the cells, indicating polar accumulation of the sensory kinases. For quantitative evaluation, line profiles of the imaged fluorescence intensities were generated; these revealed that the fluorescence intensity of the polar bright spots was 2.3-8.5 times higher than that of the cytoplasm. With respect to the cylindrical part of the membrane, the values were lower by about $40 \%$. The polar accumulation was comparable to that of methyl-accepting chemotaxis proteins (MCPs) and MCP-related proteins. The degree of DcuS-YFP localization was independent of the presence of MCP and the expression level of $d c u S-y f p$ (or DcuS concentration). The presence of effector (fumarate or citrate, respectively) increased the polar accumulation by more than $20 \%$. Cell fractionation demonstrated that polar accumulation was not related to inclusion body formation. Therefore, sensory kinases DcuS and CitA, which regulate metabolic processes without obvious polar function, exhibit polar accumulation.
\end{abstract}

\section{INTRODUCTION}

Prokaryotes sense a large number of extra- and intracellular stimuli and adapt their metabolism, cell composition and development to the external environment. Environmental stimuli are frequently perceived by two-component systems consisting of a membrane-bound sensory histidine kinase and a corresponding response regulator (Mascher et al., 2006). The perception of external stimuli induces autophosphorylation of the sensory histidine kinase, and then the phosphoryl group is transmitted to the response regulator, which triggers the cellular response mostly by gene regulation.

The $\mathrm{C}_{4}$-dicarboxylate sensor DcuS of Escherichia coli represents a typical membrane-bound histidine protein

†These authors contributed equally to this work.

Abbreviations: MCP, methyl-accepting chemotaxis protein; YFP, yellow fluorescent protein.

Supplementary data (including five movie files) are available with the online version of this paper. kinase. It contains a distinct periplasmic sensory domain for $\mathrm{C}_{4}$-dicarboxylates that is situated between two transmembrane helices (Golby et al., 1999; Zientz et al., 1998). The structure of the periplasmic domain of DcuS in the liquid state has been solved by NMR (Pappalardo et al., 2003), and the binding site for $\mathrm{C}_{4}$-dicarboxylates has been characterized by mutagenesis (Kneuper et al., 2005; Krämer et al., 2007). The second (or C-terminal) transmembrane domain is followed by the cytoplasmic transmitter domain including the histidine kinase domain. DcuS controls the expression of genes required for fumarate respiration ( $d c u B, f u m B$ and $f r d A B C D$ ) that encode the fumarate/ succinate antiporter DcuB, fumarase FumB and fumarate reductase FrdABCD (Golby et al., 1999; Janausch et al., 2002a, b; Zientz et al., 1998). Expression of $d c u B$ occurs under anaerobic conditions, and strongly depends on induction by DcuS in the presence of $\mathrm{C}_{4}$-dicarboxylates, such as fumarate or malate. Thus, expression of $d c u B-l a c Z$ reporter-gene fusions has been used to assay the functional state of DcuS in vivo (Kneuper et al., 2005; Zientz et al., 
1998). After signal perception and autophosphorylation of DcuS, the phosphorylated response regulator DcuR binds to target genes and activates their expression (Abo-Amer et al., 2004; Janausch et al., 2004). With all the attributes of a periplasmic sensory histidine kinase, the individual DcuS homodimers are assumed to function for signal perception and transmission to response regulators (Mascher et al., 2006). CitA is a citrate-specific sensor kinase of the CitAB two-component system of $E$. coli and related bacteria (Kaspar et al., 1999; Kaspar \& Bott, 2002). Because of the similarities in sequence and overall topology, both CitA and DcuS are members of the CitA family of sensory kinases.

An uneven cellular distribution of histidine kinases controlling asymmetric processes in cell division and development has been observed in Bacillus subtilis, Pseudomonas and Caulobacter (Boyd, 2000; Shapiro et al., 2002). The localization of these histidine kinases within the cell often relates to their site of function, and typically features a polar localization. The chemoreceptors of chemotaxis (Lybarger \& Maddock, 2000) show also an asymmetric distribution within E. coli cells, although no asymmetric distribution is expected for chemotaxis regulation. A uniform distribution over the cell membrane is assumed for kinases controlling metabolic processes or perceiving stimuli that are evenly distributed over the cell.
Here, the cellular distribution of DcuS and CitA, representing related sensory kinases of the CitA family, was studied in detail. The sensory kinases (DcuS and CitA) control metabolic processes (fumarate respiration and citrate fermentation, respectively) without obvious asymmetric distribution in the bacterial cell. Fusions of DcuS or CitA with a modified form of green fluorescent protein allowed careful analysis of the distribution of these kinases in the cell membrane of $E$. coli by confocal laser scanning microscopy.

\section{METHODS}

Bacteria and molecular genetic methods. E. coli $\mathrm{K}-12$ strains and plasmids used in this study are listed in Table 1. Molecular genetic methods were performed according to standard procedures (Sambrook \& Russell, 2001). Genomic DNA was isolated as previously described (Chen \& Kuo, 1993); plasmids were isolated and purified using kits (Qiagen). PCR products were purified with the Qiaquick PCR purification kit (Qiagen). E. coli strains were transformed by electroporation (Dower et al., 1988).

Construction of dcuS-yfp and citA-yfp fusions. The eyfp gene was amplified from plasmid pEYFP (ClonTech) by PCR with oligonucleotide primers gfpCfor (5'-CCATGGAGCTCAAGGGCGA$\left.3^{\prime}\right)$ and gfpCrev ( $5^{\prime}$-AGCGACCGAAGCTTAGTTGG- $\left.3^{\prime}\right)$. The PCR fragment lacking the sequence of the three $\mathrm{N}$-terminal amino acids of EYFP was cloned via the SacI and HindIII sites of the oligonucleotides

Table 1. Strains of E. coli and plasmids used in this study

\begin{tabular}{|c|c|c|}
\hline Strain or plasmid & Genotype & Reference or source \\
\hline \multicolumn{3}{|l|}{ E. coli $\mathrm{K}-12$} \\
\hline MC4100 & $\mathrm{F}^{-}$araD139 $\Delta($ argF-lac $)$ U169 rpsL150 $\Delta$ lacZ relA1 flbB530 deoC1 ptsF25 rbsR & Silhavy et al. (1984) \\
\hline MG1655 & CGSC $6300, f_{n} r^{-}, \lambda$-F-P1-sensitive & Jensen $(1993)$ \\
\hline JM109 & $\begin{array}{l}\text { recA1 supE44 endA1 hsdR17 gyrA96 relA1 thi } \Delta(\text { lac-proAB }) \mathrm{F}^{\prime}\left[\text { traD36 proAB }{ }^{+} \text {, }\right. \\
\left.\text { lacI }{ }^{\mathrm{q}} \text { lacZ } \Delta \mathrm{M} 15\right]\end{array}$ & Yanisch-Perron et al. (1985) \\
\hline IMW205 & MC4100 dcuR:: $\operatorname{Kan}^{\mathrm{r}}$ & Zientz et al. (1998) \\
\hline IMW237 & MC4100 $\lambda\left[\Phi\left(d c u B^{\prime}-^{\prime} l a c Z\right) h y b, b l a^{+}\right]$ & Kneuper et al. (2005) \\
\hline IMW260 & MC4100 $\lambda\left[\Phi\left(d c u B^{\prime}-{ }^{\prime} l a c Z\right) h y b, b l a^{+}\right] d c u S::$ Cam $^{\mathrm{r}}$ & Zientz et al. (1998) \\
\hline IMW262 & MC4100, dcuS:: $\mathrm{Cam}^{\mathrm{r}}$ & Zientz et al. (1998) \\
\hline IMW280 & MC4100 $\lambda\left[\Phi\left(d c u B^{\prime}-^{\prime} l a c Z\right) h y b, b l a^{+}\right] c i t A:: \operatorname{Kan}^{\mathrm{r}}$ & Krämer et al. (2007) \\
\hline IMW502 & MC4100 $\Delta d c u B$ & This study \\
\hline UU1250 & $\Delta t s r-7028 \Delta($ tar-tap $) 5201 \Delta \operatorname{trg}-100 \Delta a e r-1\left(\operatorname{Kan}^{\mathrm{r}}\right)$ & Ames et al. (2002) \\
\hline VS100 & $\Delta c h e Y$, in-frame $c h e Y$ deletion & Sourjik \& Berg (2000) \\
\hline \multicolumn{3}{|l|}{ Plasmids } \\
\hline pBAD30 & Expression vector; $\mathrm{pACYC}$ ori, $\mathrm{P}_{\mathrm{BAD}}$ promoter $\left(\mathrm{Ap}^{\mathrm{r}}\right)$ & Guzman et al. (1995) \\
\hline pDK108 & $\operatorname{Tar}(1-331)-Y F P$ expression plasmid; $p \operatorname{Trc} 99 a$ derivative $\left(A p^{r}\right)$ & Kentner et al. (2006) \\
\hline pET28a & Expression vector; pBR ori, T7 promoter, His-tag $\left(\operatorname{Kan}^{\mathrm{r}}\right)$ & Novagen \\
\hline pEYFP & Vector containing enhanced GFP variant YFP $\left(\mathrm{Ap}^{\mathrm{r}}\right)$ & Clontech \\
\hline pMW151 & DcuS expression plasmid; pET28a derivative $\left(\operatorname{Kan}^{r}\right)$ & Janausch et al. (2002a) \\
\hline pMW384 & DcuS-YFP expression plasmid; pMW391 derivative $\left(\operatorname{Kan}^{\mathrm{r}}\right)$ & This study \\
\hline pMW391 & 'yfp, protein fusion plasmid; pET28a derivative $\left(\operatorname{Kan}^{\mathrm{r}}\right)$ & This study \\
\hline pMW407 & DcuS-YFP expression plasmid; pBAD30 derivative $\left(\mathrm{Ap}^{\mathrm{r}}\right)$ & This study \\
\hline pMW442 & CitA-YFP expression plasmid; pBAD30 derivative $\left(\mathrm{Ap}^{\mathrm{r}}\right)$ & This study \\
\hline pMW557 & pMW442 with additional $\mathrm{Cam}^{\mathrm{r}}$ & This study \\
\hline pVS1 & CheY-YFP expression plasmid; pBAD18K derivative $\left(\operatorname{Kan}^{\mathrm{r}}\right)$ & Sourjik \& Berg (2000) \\
\hline
\end{tabular}


into pET28a (Novagen) behind the inducible T7 promoter. The resulting construct (pMW391) served as the plasmid for cloning EYFP to the C-terminal end of proteins. $d c u S$ was amplified by PCR with oligonucleotide primers dcuSpetCfor (5'-CGGCAGCCATATGAGACATTC- $\left.3^{\prime}\right)$ and dcuSpetCrev ( $5^{\prime}$-TGTTCGAGAGCTCCCCGTC-3') from pMW151 (Janausch et al., 2002a). The PCR fragment was cloned via the NdeI and SacI sites into pMW391 at the $5^{\prime}$ end of eyfp. The resulting construct (pMW384) encoded DcuS(1-539)(Lys)-EYFP(4-240) (hereafter referred to as $d c u S-y f p$ or DcuS-YFP fusion). The fusion protein also carried an $\mathrm{N}$-terminal $\mathrm{His}_{6}$-tag and a thrombin cleavage site. The $d c u S-y f p$ fusion was inserted into pBAD30 using restriction endonuclease XbaI (pMW407). CitA-YFP was constructed as described for DcuS-YFP. The citA gene was amplified from genomic DNA of E. coli MG1655 by PCR with oligonucleotide primers citApetCfor (5'-CAACCCGCTAGCAAAACAATG- $3^{\prime}$ ) and citApetCrev (5'-CGTCCTCAGAGCTCAATAGG-3') and by cloning the PCR fragment via NheI and SacI sites into pMW391. The citA-yfp fusion was subsequently inserted into pBAD30 via XbaI (pMW442). The sequences of the resulting constructs were verified by DNA sequencing.

Growth and expression. For microscopy, cell fractionation and Western blot analysis, bacteria were grown aerobically in LB medium at 30,33 , or $37^{\circ} \mathrm{C}$ for $3-4.5 \mathrm{~h}$, corresponding to the mid-exponential phase of growth (Sambrook \& Russell, 2001). E. coli strains IMW262/ pMW407, JM109/pMW407, UU1250/pMW407 and JM109/pMW442 were induced from the beginning of incubation with $10-333 \mu \mathrm{M} \mathrm{L}$ arabinose. Ampicillin was added to a concentration of $100 \mu \mathrm{g} \mathrm{ml}^{-1}$. The reference strains VS100/pVS1 and JM109/pDK108 were grown aerobically in LB broth for $4 \mathrm{~h}$ at $33{ }^{\circ} \mathrm{C}$ in the presence of $333 \mu \mathrm{M}$ arabinose and $50 \mu \mathrm{g}$ kanamycin $\mathrm{ml}^{-1}$, or $1 \mathrm{mM}$ IPTG and $100 \mu \mathrm{g}$ ampicillin $\mathrm{ml}^{-1}$, respectively.

Cell fractionation. Arabinose-induced bacteria were harvested by centrifugation at $6300 \mathrm{~g}$ for $10 \mathrm{~min}$. All further steps were done at $4{ }^{\circ} \mathrm{C}$. Washed cells from $400 \mathrm{ml}$ medium were resuspended in $8 \mathrm{ml}$ buffer (50 mM Tris/ $\mathrm{HCl}, \mathrm{pH} 7.7,10 \mathrm{mM} \mathrm{MgCl}_{2}$ ). The bacteria were broken using a French press (3 times at $8274 \mathrm{kPa}$ ) and then centrifuged at $8600 \mathrm{~g}$ for $10 \mathrm{~min}$ to sediment debris and potential inclusion bodies (low-speed pellet) and to separate them from the soluble fraction and cytoplasmic membranes contained in the supernatant (low-speed supernatant). For (semi-dry) Western blot analysis, proteins from the low-speed pellet and the low-speed supernatant were separated by SDS-PAGE and transferred to nitrocellulose membranes as described by Towbin et al. (1979). Membranes were treated with mouse polyclonal antibodies (Qiagen) against the $\mathrm{His}_{6}$-tag of the fusion protein. Protein bands were detected with secondary IgG antibodies coupled to peroxidase (Sigma-Aldrich). Protein bands of three independent preparations were visualized and quantified by using the Kodak Image Station $440 \mathrm{CF}$ and the Kodak Molecular Imaging software.

To sediment the membrane fraction, the low-speed supernatant was centrifuged at $200000 \mathrm{~g}$ for $65 \mathrm{~min}$. The membrane pellet was washed twice ( $1 \mathrm{mM}$ Tris/HCl, pH 7.7, $3 \mathrm{mM}$ EDTA), homogenized in buffer (50 mM Tris/HCl, pH 7.7, $10 \%, w / v$, glycerol), and solubilized by the addition of Empigen BB $(30 \%, w / v$, Calbiochem) to a final concentration of $2 \%(\mathrm{w} / \mathrm{v})$ as described by Janausch et al. (2002a).

The low-speed supernatant fraction was fractionated in a sucrose step-gradient $(0 \%, 10 \%$ and $40 \%$ sucrose) by ultracentrifugation at $200000 \mathrm{~g}$ for $2 \mathrm{~h}$. Fractions of $1 \mathrm{ml}$ were collected and $20 \mu \mathrm{l}$ of each fraction was subjected to SDS-PAGE to examine banding of DcuSYFP in the protein/membrane fraction and in the protein-aggregate fraction. Isolated and purified DcuS-YFP was used as a standard.

Western blot analysis of DcuS-YFP expression levels. E. coli cells induced by different arabinose concentrations were sedimented by centrifugation and dissolved in SDS-containing sample buffer (Laemmli, 1970). The dissolved whole-cell proteins were separated by SDS-PAGE and transferred to nitrocellulose membranes (Towbin et al., 1979). Membranes were treated with rabbit polyclonal antiserum (Eurogentec) raised against the periplasmic domain of DcuS and detected with secondary IgG antibodies coupled to peroxidase (Sigma-Aldrich).

Test for DcuS and CitA function in vivo. E. coli IMW260 $\left(d c u S:: \mathrm{Cam}^{\mathrm{r}}, \lambda\left[\Phi d c u B^{\prime}--^{\prime}\right.\right.$ lacZ) $\left.]\right)$ containing plasmids with various forms of $d c u S$ as indicated was grown anaerobically at $37{ }^{\circ} \mathrm{C}$ in $\mathrm{M} 9$ mineral medium (Miller, 1992) with glycerol (50 mM), DMSO $(20 \mathrm{mM})$ and fumarate $(20 \mathrm{mM})$ as the inducer. Samples were withdrawn at $\mathrm{OD}_{578} 0.5-0.7$ for measurement of $\beta$-galactosidase activity. For testing CitA-YFP function, strain IMW280 (citA:: $\mathrm{Kan}^{\mathrm{r}}$, $\left.\lambda\left[\Phi d c u B^{\prime}-{ }^{\prime} l a c Z\right)\right]$ ) was used, where expression of $d c u B^{\prime}-{ }^{\prime} l a c Z$ responds to the presence of functional CitA (Krämer et al., 2007). The bacteria with or without plasmid pMW557 (citA-yfp in pBAD30, $\mathrm{Cam}^{\mathrm{r}}$ ) were grown as described for strain IMW260, but with citrate $(20 \mathrm{mM})$ as the inducer.

Spectroscopy. Absorption and fluorescence spectra were measured in $1 \mathrm{ml}$ semi-microcuvettes with a Bruins Instruments Omega 20 spectrophotometer and a Jobin-Yvon Spex FluoroMax-2 spectrofluorimeter. Fluorescence spectra were corrected for the wavelength dependence of the fluorimeter and the inner filter effect.

Microscopy. For microscopy, E. coli cells were harvested, immobilized with poly-lysine films on backed coverslides, and washed with PBS buffer. All measurements were made in PBS buffer under ambient conditions using two different confocal fluorescence microscopes. On the one hand, a custom-built microscope (based on a Zeiss Axiovert 135 TV inverted microscope) was used, which has single-molecule sensitivity (Erker et al., 2005; Kulzer et al., 1999). Images with a typical size of $10 \mu \mathrm{m} \times 10 \mu \mathrm{m}$ and $128 \times 128$ pixels were recorded with an integration time of $5 \mathrm{~ms}$ per pixel (objective: Zeiss Plan-Neofluar $100 \times / 1.30$ oil). An argon-ion laser operating at $488 \mathrm{~nm}$ attenuated to a power of $0.5 \mu \mathrm{W}$ was used for excitation. Emission was split into two beams (50:50), which simultaneously allowed the detection of total intensity (APD: Perkin-Elmer SPCMAQR-14) and the recording of fluorescence spectra (spectrograph, Acton Spectra Pro-300i; Peltier-cooled CCD-camera, LaVision IM3 QE CAM). Images were recorded by moving the sample via a $3 \mathrm{D}$ piezoscanner (Physik Instrumente P-731.20 and P-721.CLQ PIFOC). Alternatively, a commercial confocal microscope (Leica DM IRE2) with an argon-ion laser $(488 \mathrm{~nm}, 0.4 \mu \mathrm{W})$ was used. The recorded images were typically $11.72 \mu \mathrm{m} \times 11.72 \mu \mathrm{m}$ in size with a resolution of $512 \times 512$ pixels (objective: Leica HCX PL APO $40 \times / 1.25-0.75$ oil).

The cellular brightness distributions of individual cells were analysed by line scans and 3D-reconstruction using self-written software based on Igor Pro (Wavemetrics). For 3D-reconstruction, a stack of images was recorded for each cell. The images differed with respect to focus position along the optical axis. Typical $z$-increments were in the range of $150-200 \mathrm{~nm}$.

\section{RESULTS}

\section{Functional state of DcuS-YFP and CitA-YFP fusion proteins}

The $d c u S$ gene of $E$. coli encoding the $\mathrm{C}_{4}$-dicarboxylate sensory histidine kinase DcuS was fused to the $y f p$ gene encoding the enhanced yellow fluorescent variant (YFP) of green fluorescent protein (GFP). For cloning, a low-copy 
plasmid (pBAD30) was used to ensure low- or moderatelevel expression of the cloned gene (Guzman et al., 1995). The plasmid-borne $d c u S^{\prime}-^{\prime} y f p$ fusion encoding DcuS-YFP complemented anaerobic growth of a DcuS-deleted E. coli strain (IMW260) in the presence of glycerol and fumarate. In addition, the plasmid restored expression of the $d c u B^{\prime}-$ ' lacZ reporter gene fusion in the presence of fumarate to a level approximately the same as that obtained with a plasmid carrying wild-type $d c u S$ (Table 2). Both findings demonstrate that DcuS is functional upon fusion with YFP. The fluorescence properties of the bacteria containing DcuS-YFP were tested by fluorescence spectroscopy, which showed spectral characteristics for YFP fusion proteins (see supplementary data). Therefore, DcuS and YFP were both functional in the fusion protein. To ensure that the fluorescence was derived from membrane-integral DcuSYFP, the presence of DcuS-YFP inclusion bodies and their contribution to the fluorescence of the cells was measured. Sucrose step-gradient centrifugation (not shown), cell fractionation and Western blot analysis showed that less than $32 \%$ of DcuS-YFP protein and maximally $10 \%$ of the total fluorescence of the cells resided in inclusion bodies (Table 3). Based on the small contribution of the inclusion bodies $(<10 \%)$, it can be concluded that the fluorescence in the bacteria corresponds to membrane-integral DcuS-YFP.

The functional state of CitA-YFP was confirmed in a similar way as for DcuS. The plasmid encoding CitA-YFP restored the function of CitA in a CitA-deleted E. coli strain (IMW280) to more than $90 \%$ in the stimulation of $d c u B^{\prime}-$ ' lacZ in the presence of citrate (not shown). Therefore CitA-YFP can be regarded as functional as well.

\section{Fluorescence microscopy of single cells}

The cellular distributions of the fusion proteins within $E$. coli were studied by live imaging of immobilized cells in buffer using confocal scanning fluorescence microscopy. Two different microscopes were used in order to reveal different aspects of the samples (see Methods). The custom-built microscope allowed synchronous recording of images and emission spectra (e.g. Supplementary Fig.

Table 2. Functional test of DcuS-YFP by induction of $d c u B^{\prime}-$ 'lacZ expression

E. coli IMW260 [MC4100 dcuS: : $\mathrm{Cam}^{\mathrm{r}}, \lambda\left(\Phi d c u B^{\prime}-{ }^{\prime}\right.$ lacZ $\left.)\right]$ was grown anaerobically in M9 medium containing glycerol and DMSO as growth substrates with and without fumarate $(20 \mathrm{mM})$ as effector.

\begin{tabular}{|c|c|c|}
\hline \multirow[t]{2}{*}{ Strain } & \multicolumn{2}{|c|}{$\begin{array}{c}\text { Expression of } d c u B^{\prime}--^{\prime} l a c Z \\
\text { (Miller units) }\end{array}$} \\
\hline & + Fumarate & - Fumarate \\
\hline IMW260 $\left(d c u S^{-}\right)$ & 5 & 8 \\
\hline IMW260 (pMW151; dcuS $\left.{ }^{+}\right)$ & 279 & 2 \\
\hline IMW260 (pMW384; dcuS-yfp) & 332 & 9 \\
\hline
\end{tabular}

Table 3. Distribution of DcuS-YFP fusion protein and fluorescence in cellular compartments of $E$. coli

The cell homogenate of E. coli IMW262/pMW407 (dcuS ${ }^{-}$) induced with $333 \mu \mathrm{M}$ arabinose for $4 \mathrm{~h}$ was separated into supernatant and pellet fraction by low-speed centrifugation. Quantitative Western blot analysis (mean of three independent preparations) was performed with both fractions. Fluorescence of low-speed supernatant (soluble proteins and membranes) and low-speed pellet fraction (debris and inclusion bodies) was detected after excitation at $488 \mathrm{~nm}$.

\begin{tabular}{|lcc|}
\hline Fraction & Western blot & Fluorescence \\
\hline Low-speed supernatant & $68 \%$ & $90 \%$ \\
Low-speed pellet & $32 \%$ & $10 \%$ \\
\hline
\end{tabular}

S1) and was more sensitive (Supplementary Fig. S2). The commercial microscope was much faster and allowed the recording of more images per time interval, resulting in higher statistical significance.

In various E. coli wild-type strains expressing DcuS-YFP, the fluorescence intensity of most of the cells was not homogeneously distributed; instead, there were bright spots near the cell poles (Fig. 1, upper row). The individual cells showed spots at the poles that were much brighter than the fluorescence intensity at the membrane of the cylindrical part of the cell, and the fluorescence at the membrane was brighter than that in the cytoplasmic region. For quantitative evaluation of the fluorescence intensity in E. coli cells, line profiles were generated (lower row of Fig. 1) to reflect the fluorescence intensities of the cells along the lines indicated in the images of the upper row of Fig. 1. The line profile of the cell containing DcuS-YFP showed two (polar) peaks corresponding to the bright spots at the cell poles. The ratio of polar maximum to cytoplasm fluorescence intensity (ratio polar maximum/cytoplasm) was 6.9 for the first (polar) peak and 3.6 for the second one. For the same (polar) peaks, the ratios of polar maximum to the cylindrical part of the cell membrane (ratio polar maximum/membrane) were 4.3 and 2.2, respectively. The latter value might reflect the situation of membrane proteins more directly. For other proteins with polar localization mostly the former value is quoted (Liberman et al., 2004). Therefore, for reasons of comparability the former ratio (polar maximum/ cytoplasm) will be used here as well.

The line profiles of most investigated cells revealed peaks at both cell poles. The fluorescence ratios (polar maximum/ cytoplasm) of the brighter polar spots were typically in the range of 2.5-8.5 for the custom-built microscope (Supplementary Fig. S2) and in the range of 2.6-3.6 when measured with the commercial microscope (Table 4). For the data presented in Table 4, no preselection of cells was made, but all cells which gave a clear image were analysed. Heterogeneity of the analysed cells is represented by the histograms shown in Supplementary Fig. S3. Each value in Table 4 corresponds to the mean value of one of the histograms in Supplementary Fig S3. 3D reconstruction of 

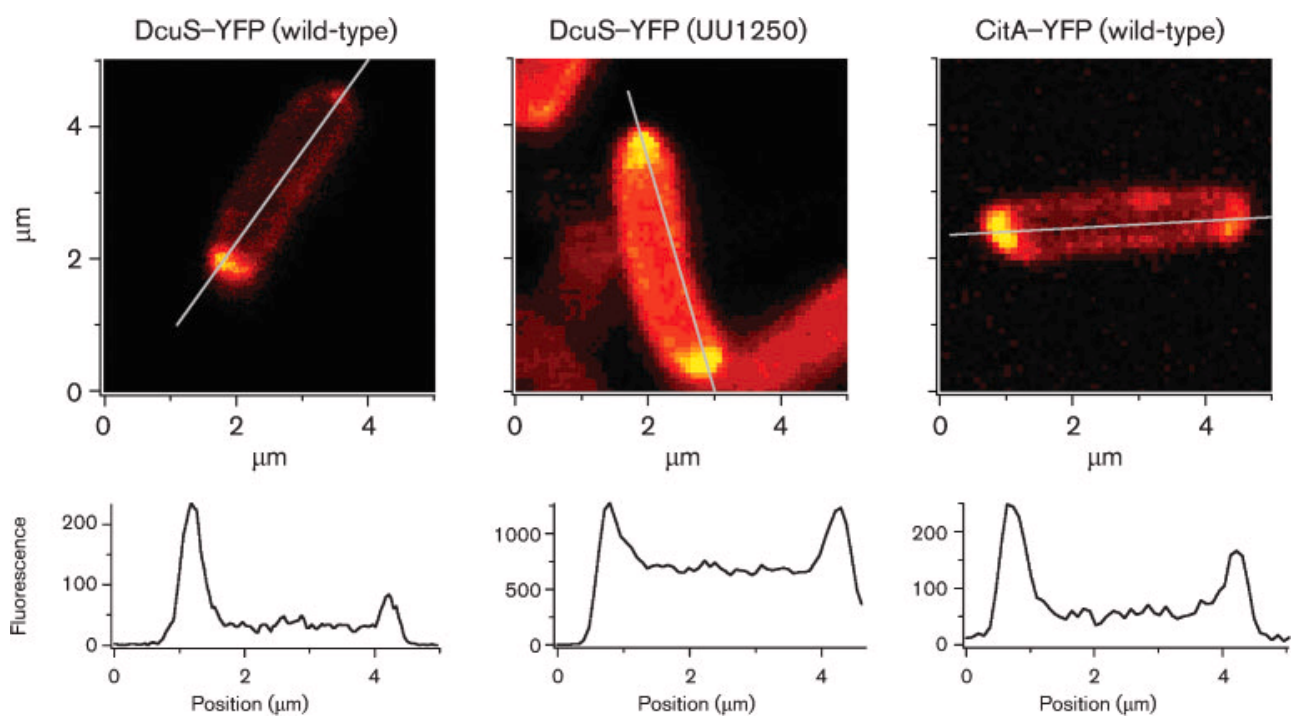

Fig. 1. Localization of DcuS-YFP and CitA-YFP in E. coli. Upper row: single E. coli cells expressing DcuS-YFP (strain JM109/pMW407 and UU1250/pMW407) or CitA-YFP (strain JM109/pMW442) fusion protein recorded with a custom-built confocal microscope. Lower row: fluorescence-intensity profiles along the lines indicated in the images above.

individual cells confirmed the polar localization of DcuSYFP (Supplementary data, movies 1-4).

Polar accumulation of the DcuS-YFP fusion protein was observed to the same extent not only in the DcuS-deficient strain (IMW262), but in the CheY-deleted strain (VS100) as well (data not shown). A similar result was observed in the chemoreceptor-deleted strain (UU1250) (Fig. 1), in which the five genes encoding the methyl-accepting chemotaxis protein (MCP) complexes had been deleted. DcuS-YFP also accumulated at the cell poles in strain UU1250, but the overall fluorescence intensity of the cells in this strain was, on average, 2.5-fold higher than that in the other strains (see line profiles).

The localization of CitA, a sensor kinase closely related to DcuS (Kaspar \& Bott, 2002; Mascher et al., 2006), was investigated in the same way. Again, a C-terminal fusion with YFP was constructed (CitA-YFP) and its cellular localization was studied by fluorescence microscopy. The localization of CitA (Fig. 1) was the same as that of DcuS, i.e. the fusion protein was detected in the cell membrane and accumulated at the cell poles. The mean ratio (polar maximum/cytoplasm) obtained with the commercial microscope was in the range of 3.5-5.1 for CitA-YFP (Table 4).

\section{Localization of DcuS-YFP and CitA-YFP versus proteins with polar, membranous or cytoplasmic localization}

As a reference for a protein with known polar accumulation, CheY-YFP was tested. CheY is a water-soluble intracellular protein that associates with the MCPs, which are localized at the cell poles. The polar localization of $\mathrm{CheY}$ is comparable with that of the MCPs (Sourjik \& Berg, 2000). Images and

Table 4. Influence of effectors fumarate and citrate on the polar accumulation of DcuS-YFP and CitA-YFP

Bacteria containing pMW407 expressing DcuS-YFP or pMW442 expressing CitA-YFP were grown at $30{ }^{\circ} \mathrm{C}$ for $4 \mathrm{~h}$ with $133 \mu \mathrm{M}$ arabinose in the presence or absence of effector $(20 \mathrm{mM}$ of fumarate or citrate, respectively). Each value in the table represents the mean \pm SD of the ratio (polar maximum/cytoplasmic fluorescence intensity) obtained from different cells (cell number $n$ ) using the commercial microscope. The corresponding histograms are plotted in Supplementary Fig. S3. No preselection was made but all cells which gave a clear image were analysed.

\begin{tabular}{|lcccc|}
\hline Protein & $\begin{array}{c}\text { Strain (relevant } \\
\text { chromosomal genotype) }\end{array}$ & $\begin{array}{c}\text { Mean ratio } \\
\text { with effector }\end{array}$ & $\begin{array}{c}\text { Mean ratio } \\
\text { without effector }\end{array}$ & $\begin{array}{c}n \text { with/without } \\
\text { effector }\end{array}$ \\
\hline DcuS-YFP & Wild-type & $3.2 \pm 0.2$ & $2.6 \pm 0.2$ & 2.2 \\
& IMW262 $\left(d c u S^{-}\right)$ & $3.4 \pm 0.1$ & $2.6 \pm 0.1$ & $12.7 \pm 0.1$ \\
& IMW502 $\left(d c u B^{-}\right)$ & $3.6 \pm 0.1$ & $2.7 \pm 0.1$ & $102 / 115$ \\
CitA-YFP & IMW205 $\left(d c u R^{-}\right)$ & $2.9 \pm 0.1$ & $3.5 \pm 0.2$ & $101 / 67$ \\
& JM109 (wild-type) & $5.1 \pm 0.2$ & & $129 / 124$ \\
\hline
\end{tabular}


line profiles of CheY-YFP (Fig. 2) showed the polar accumulation of this protein. The fluorescence intensity at the poles was three times higher than the intracellular level. Thus CheY, DcuS and CitA show polar accumulation to similar extents. Slight differences in the polar accumulation could be due to some differences in genetic background of the strains used for this experiments. As a control for a protein with an even distribution over the cell membrane, a YFP fusion with truncated $\operatorname{Tar}(1-331)$ was used. Tar is a membrane-bound chemoreceptor which localizes at the cell poles, whereas the C-terminal truncated $\operatorname{Tar}(1-331)$ is membrane bound, but without polar accumulation (Kentner et al., 2006). Images of cells expressing the truncated $\operatorname{Tar}(1-331)$ fused to YFP revealed that fluorescence was symmetrically distributed over the entire cell membrane (Fig. 2). As a cytoplasmic protein, wild-type YFP was used for comparison. Water-soluble YFP was homogeneously distributed in the cytoplasm, resulting in a line profile of roughly constant fluorescence level within the cell but with lower fluorescence at the membranes (Fig. 2). E. coli cells expressing no YFP variants produced only autofluorescence, with an intensity 10-fold lower than that of cells expressing YFP fusions (see line profiles). This autofluorescence resulted in relatively weak but not specifically localized fluorescence within the cells (Fig. 2).

\section{Polar accumulation of DcuS-YFP is independent of DcuS expression rate and time}

Expression of the DcuS-YFP fusion protein was induced by various concentrations of arabinose. The pBAD30 vector is generally used for moderate-level expression due to its low copy number. After induction with 133 or $333 \mu \mathrm{M}$ arabinose, high expression of DcuS-YFP was detected by Western blotting with anti-DcuS antiserum (Fig. 3). In the presence of $10 \mu \mathrm{M}$ arabinose, the expression of DcuS-YFP sharply decreased by a factor of 10 but was still significantly higher than that of the endogenous DcuS. Compared to the endogenous DcuS expression level (maximally $0.004 \%$ of the cell protein), the amounts of DcuS-YFP induced by 10 , 133 or $333 \mu \mathrm{M}$ arabinose were estimated to be about $0.04 \%, 0.4 \%$ or $0.4 \%$, respectively, of the cell protein. The further localization studies were performed with cells induced by arabinose concentrations as low as $10 \mu \mathrm{M}$, and up to $333 \mu \mathrm{M}$ arabinose for other experiments. With the induction by $10 \mu \mathrm{M}$ arabinose, measurements could be performed at a low DcuS level as close as possible to the in vivo conditions, but at the fluorescence detection limit. On the other hand, induction by $333 \mu \mathrm{M}$ arabinose resulted in DcuS levels which were higher than the endogenous levels, but the inducer concentration was still much lower than that commonly used for localization studies.

Concentration dependence of arabinose induction and time-course of polar accumulation of DcuS-YFP was studied. The influence of inducer concentration on DcuSYFP localization was studied at the single-cell level. The fluorescence intensity ratios, (polar maximum/cytoplasm) and (polar maximum/membrane), were plotted as a function of the arabinose concentration used for fusionprotein expression (Fig. 4). Despite some fluctuations in the two ratios, polar accumulation of DcuS-YFP not only already appeared at low concentrations of both inducer and
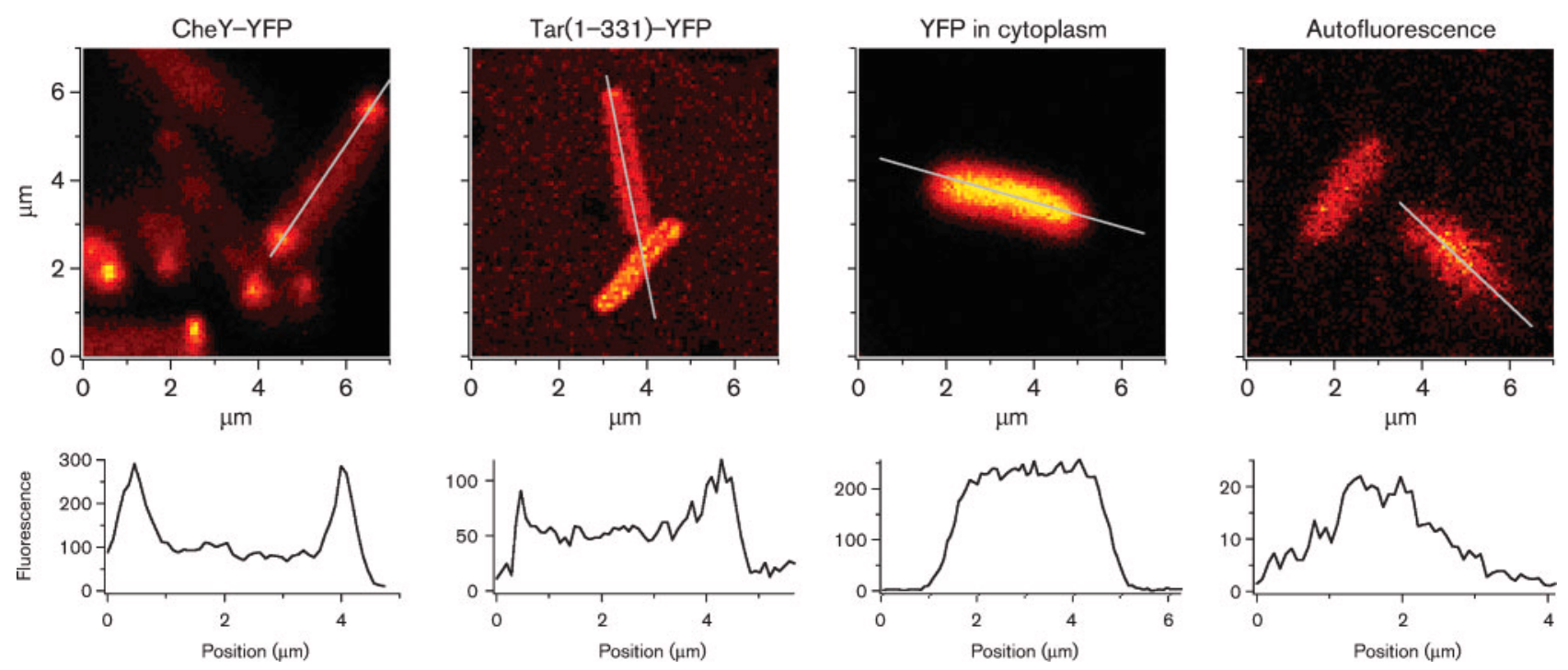

Fig. 2. Controls for proteins with polar, membranous and cytoplasmic localization in E. coli. Upper row: images of single $E$. coli cells expressing CheY-YFP (strain VS100/pVS1) or Tar(1-331)-YFP (strain JM109/pDK108) fusion proteins, wild-type YFP (JM109/pEYFP), or no YFP (JM109). The image of Tar(1-331)-YFP was recorded with the commercial microscope, the other images with the custom-built microscope. Lower row: fluorescence-intensity profiles along the lines indicated in the images above. 


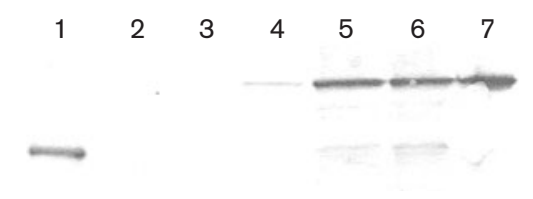

Fig. 3. Detection of DcuS-YFP in lysed cells of $E$. coli by Western blotting with antiserum against the periplasmic domain of DcuS. E. coli JM109/pMW407 was grown under aerobic conditions in LB broth in the presence of $0 \mu \mathrm{M}$ (lane 3), $10 \mu \mathrm{M}$ (lane 4), $133 \mu \mathrm{M}$ (lane 5) and $333 \mu \mathrm{M}$ (lane 6) arabinose. For lane 2, E. coli wild-type (JM109) was grown anaerobically in M9 mineral medium with $50 \mathrm{mM}$ glycerol and $20 \mathrm{mM}$ fumarate. Cells were sedimented, the pellet was dissolved in SDS sample buffer, and $60 \mu \mathrm{g}$ (lanes 3-6) or $120 \mu \mathrm{g}$ (lane 2) of cell lysates were subjected to SDS-PAGE. After blotting onto a nitrocellulose membrane, DcuS and DcuS-YFP were detected by immunostaining with antiserum against the periplasmic domain of DcuS. Lanes 1 and 7 contain $1 \mu \mathrm{g}$ of purified DcuS or DcuS-YFP, respectively.

DcuS-YFP, but remained constant at higher concentrations of both inducer and DcuS-YFP. Therefore polar accumulation of DcuS-YFP is independent of the expression level.

The time-course of DcuS-YFP and CitA-YFP localization was also investigated. After induction of DcuS-YFP or CitA-YFP expression from the beginning of incubation, cell samples were collected every $30 \mathrm{~min}$ and analysed for polar localization of the fusion proteins at the single-cell level. The maximum fluorescence intensity per cell and the ratio (polar maximum/cytoplasm) are plotted in Fig. 5. DcuS-YFP and CitA-YFP behaved very similarly with respect to their fluorescence-intensity kinetics (Fig. 5a). After a lag phase of about $1.5-2 \mathrm{~h}$, fluorescence intensity

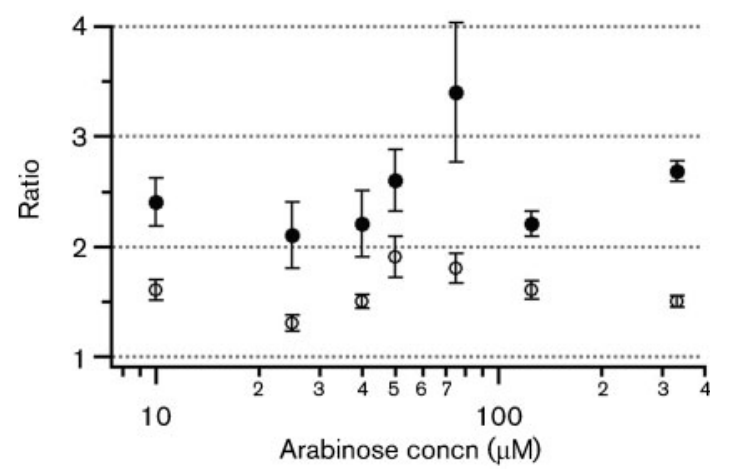

Fig. 4. Dependence of DcuS localization on expression by various concentrations of inducer (arabinose). Expression of the DcuSYFP fusion protein (governed by an araBAD promoter) in $E$. coli (strain IMW262/pMW407) was induced with increasing concentrations of arabinose. Samples were harvested after $4.5 \mathrm{~h}$. Single cells were imaged with the commercial confocal microscope. The intensity ratios (polar maximum/cytoplasm, - ) and (polar maximum/membrane, $\bigcirc$ ) are plotted (means \pm SD of 3-28 cells).
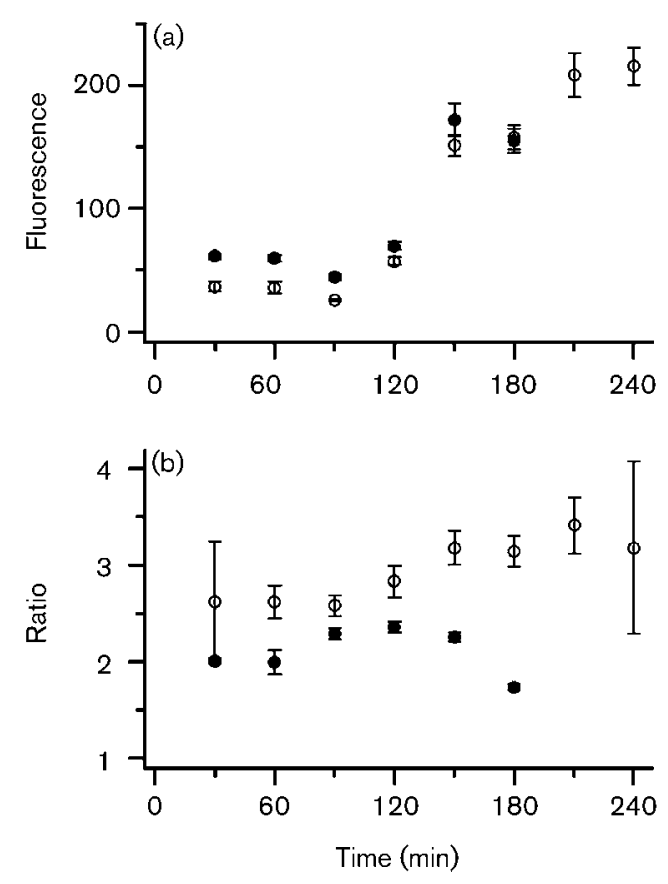

Fig. 5. Dependence of localization on the time after induction of fusion protein expression. Expression of the fusion protein (DcuSYFP, strain IMW262/pMW407, - C CitA-YFP, strain JM109/ pMW442, $\bigcirc)$ in $E$. coli was induced with $333 \mu \mathrm{M}$ arabinose. Samples were collected every $30 \mathrm{~min}$ and single cells were imaged with the commercial confocal microscope. The means \pm SD of 5-39 cells are plotted. (a) Peak fluorescence intensity of the bright spots within the cells; (b) intensity ratio (polar maximum/ cytoplasm).

increased continuously. By contrast, with respect to their ratio kinetics, the two proteins behaved differently (Fig. 5b). The ratio for DcuS-YFP was close to 2.0 after $30 \mathrm{~min}$ of induction and persisted for at least $2 \mathrm{~h}$. The ratio for CitAYFP was 2.5 after $30 \mathrm{~min}$ and increased with time. Despite the different behaviour of their ratio kinetics, the polar accumulation of both DcuS-YFP and CitA-YFP was established already within the first $30 \mathrm{~min}$ after their expression, and their localization persisted for several hours.

\section{Polar accumulation responds to the presence of effector}

Polar accumulation of DcuS-YFP and CitA-YFP was studied in the presence or absence of the corresponding effectors (fumarate or citrate, respectively). The ratios (polar maximum/cytoplasm) of individual cells from independent cultures were averaged (Table 4 and Supplementary Fig. S3) and analysed by Welch's $t$-test (test hypothesis: the average does not change upon adding effector, significance level of $5 \%$ ). The DcuS-YFP fusion protein in the DcuS deleted strain (IMW262) revealed a significant increase in polar accumulation upon the presence of the effector, fumarate. The same increase in 
polar accumulation of DcuS-YFP was observed for both the DcuB-deleted strain (IMW502) and wild-type strain (MC4100). No increase in polar accumulation of DcuSYFP was observed in the presence of acetate (data not shown), suggesting that the effect of fumarate on polar accumulation of DcuS-YFP is specific. In the DcuR-deleted strain (IMW205), the polar accumulation of DcuS-YFP increased only to a very low extent in the presence of fumarate. Taken together, the data suggest that presence of fumarate increases the polar accumulation of DcuS-YFP, and the presence of DcuR is required for this effect.

The polar accumulation of CitA-YFP significantly increased upon addition of citrate to the wild-type strain JM109, but no increase in polar accumulation of CitA-YFP was observed in the presence of fumarate (data not shown), suggesting that the effect of citrate on polar accumulation of CitA-YFP is specific.

\section{DISCUSSION}

\section{DcuS-YFP and CitA-YFP accumulate at the cell poles of $E$. coli}

The cellular localization of DcuS and CitA after fusion with YFP was studied. DcuS-YFP and CitA-YFP accumulated at one or both cell poles of E. coli, and the polar accumulation depended solely on the DcuS or CitA proteins, whereas YFP contributed neither to membranous nor to polar localization. The expression of recombinant proteins may result in the formation of inclusion bodies, which are frequently located close to the cell poles (Margolin, 2000), thus mimicking the polar localization of proteins. The potential artefact of inclusion bodies was excluded for DcuS-YFP by the following experiments. (i) After cells were fractioned, the fraction potentially containing inclusion bodies showed only a low fluorescence intensity (at most $10 \%$ of the total). (ii) DcuS localization was found to be independent of the inducer (arabinose) concentration controlling $d c u S-y f p$ expression, and therefore also independent of the protein level. (iii) Polar accumulation of DcuS-YFP or CitA-YFP became evident soon after induction at low levels of expression (Fig. 5b), implying that polar accumulation of DcuS-YFP or CitA-YFP was not due to overexpression of either fusion protein. Points (ii) and (iii) also argue against the formation of non-specific aggregates in the membrane. Altogether, the polar accumulation of DcuS and CitA appears to represent the native site of the kinases.

Polar accumulation can be quantified by a fluorescence intensity ratio (polar maximum/cytoplasm). The ratio for the polar accumulation of either DcuS-YFP or CitA-YFP was 2.5 or above, implying that these proteins accumulated but were not located exclusively at the cell poles.

\section{Factors affecting polar accumulation of DcuS- YFP and CitA-YFP}

The polar accumulation of DcuS-YFP and CitA-YFP was significantly increased in the presence of effector (fumarate or citrate). In the case of DcuS, the increase depended on the presence of the related response regulator DcuR, suggesting that the polar accumulation is related to the functional state of the DcuSR two-component system. Both the polar accumulation at low concentrations of DcuS and its further increase in the presence of the effector fumarate could indicate that the polar accumulation is of physiological relevance, and that DcuR might play a role in DcuS localization. However, the mechanistic background for the supposed role of DcuR in polar accumulation of DcuS is not clear.

\section{Comparison of DcuS accumulation with that of other localized proteins}

MCPs represent the paradigm for polar-localized (or accumulated) sensory proteins (Lybarger \& Maddock, 2000; Maddock \& Shapiro, 1993; Sourjik \& Berg, 2000). $\mathrm{MCP}$-associated proteins, such as $\mathrm{CheY}$, also accumulate at the cell poles to a similar extent. The fluorescence ratios (polar maximum/cytoplasm) for MCP-related proteins were reported to be in the range of 1.2-2.2, and for CheY about 1.6 (Sourjik \& Berg, 2000). In the present study, fluorescence ratios (polar maximum/cytoplasm) in the range of 2.6-3.6 (Table 4) were determined for DcuS, and a similar range was observed for CitA. Thus, the amount of DcuS that accumulated at the cell poles was comparable to that of the chemotaxis proteins even if the methods to determine the ratios were slightly different in the two studies. However, the polar accumulation of DcuS did not depend on the presence of the MCP complex. In the absence of MCP (strain UU1250), DcuS still accumulated at the cell poles (Fig. 1); hence, there seems to be no direct interaction between DcuS and MCP.

So far, polar accumulation of sensory kinases has been shown for two-component systems that control reactions with an uneven or a polar distribution, such as the segregation of cell components in symmetric (Boyd, 2000) or asymmetric cell division and development (Jensen et al., 2002; Shapiro et al., 2002; Wheeler \& Shapiro, 1999; Wingrove \& Gober, 1996). In these examples, histidine kinases often localize at the sites of their regulated processes or their partners. As shown here for DcuS and CitA, polar or asymmetric localization can be found also for sensory histidine kinases that control metabolic processes without known or predicted asymmetric distribution in the cell. It is possible that such polar accumulation is not restricted to DcuS and CitA.

For DcuS and CitA, the degree of polar accumulation increased in the presence of the corresponding effector (fumarate or citrate, respectively). This could indicate that the polar accumulation is functionally relevant and that it supports proper function of the two sensor kinases in an unknown way. Clusters of chemotaxis sensors together with the sensor-related proteins CheA and CheW are well documented (Lybarger \& Maddock, 2000). The clusters are believed to support stimulus integration and the sensitivity of 
the sensors (Sourjik, 2004; Thiem et al., 2007). However, chemotaxis requires no asymmetric distribution for the function of the sensory complexes, and the role of polar localization of the complexes is not understood. It could be related to the diffusion restriction of cell envelope proteins in the polar region (de Pedro et al., 2004), which might extend to the cytoplasmic membranes. Furthermore, polar localization could be affected by phospholipids, e.g. in cardiolipin domains (Matsumoto et al., 2006). Consequently, it has been suggested that membrane proteins can be trapped in lipid patches of specific composition due to increased solubility. Such a mechanism for polar accumulation is also feasible for DcuS and CitA.

\section{ACKNOWLEDGEMENTS}

We are grateful to Dr V. Sourjik (ZMBH, Heidelberg, Germany) for supplying strains VS100 and UU1250 and plasmids pBAD30, pVS1 and pDK108, and to Dr A. Kleefeld (Mainz) for strain IMW502. We thank the Deutsche Forschungsgemeinschaft for financial support.

\section{REFERENCES}

Abo-Amer, A. E., Munn, J., Jackson, K., Aktas, M., Golby, P., Kelly, D. J. \& Andrews, S. C. (2004). DNA interaction and phosphotransfer of the $\mathrm{C}_{4}$-dicarboxylate-responsive DcuS-DcuR two-component regulatory system from Escherichia coli. J Bacteriol 186, 1879-1889.

Ames, P., Studdert, C. A., Reiser, R. H. \& Parkinson, J. S. (2002). Collaborative signaling by mixed chemoreceptor teams in Escherichia coli. Proc Natl Acad Sci U S A 99, 7060-7065.

Boyd, J. M. (2000). Localization of the histidine kinase PilS to the poles of Pseudomonas aeruginosa and identification of a localization domain. Mol Microbiol 36, 153-162.

Chen, W. P. \& Kuo, T. T. (1993). A simple and rapid method for the preparation of gram-negative bacterial genomic DNA. Nucleic Acids Res 21, 2260.

de Pedro, M. A., Grunfelder, C. G. \& Schwarz, H. (2004). Restricted mobility of cell surface proteins in the polar regions of Escherichia coli. J Bacteriol 186, 2594-2602.

Dower, W. J., Miller, J. F. \& Ragsdale, C. W. (1988). High efficiency transformation of E. coli by high voltage electroporation. Nucleic Acids Res 16, 6127-6145.

Erker, W., Sdorra, S. \& Basché, T. (2005). Detection of single oxygen molecules with fluorescence-labeled hemocyanins. J Am Chem Soc 127, 14532-14533.

Golby, P., Davies, S., Kelly, D. J., Guest, J. R. \& Andrews, S. C. (1999), Identification and characterization of a two-component sensor-kinase and response-regulator system (DcuS-DcuR) controlling gene expression in response to $\mathrm{C}_{4}$-dicarboxylates in Escherichia coli. J Bacteriol 181, 1238-1248.

Guzman, L. M., Belin, D., Carson, M. J. \& Beckwith, J. (1995). Tight regulation, modulation, and high-level expression by vectors containing the arabinose PBAD promoter. J Bacteriol 177, 4121-4130.

Janausch, I. G., Garcia-Moreno, I. \& Unden, G. (2002a). Function of DcuS from Escherichia coli as a fumarate-stimulated histidine protein kinase in vitro. J Biol Chem 277, 39809-39814.

Janausch, I. G., Zientz, E., Tran, Q. H., Kröger, A. \& Unden, G. (2002b). $\mathrm{C}_{4}$-dicarboxylate carriers and sensors in bacteria. Biochim Biophys Acta 1553, 39-56.
Janausch, I. G., Garcia-Moreno, I., Lehnen, D., Zeuner, Y. \& Unden, G. (2004). Phosphorylation and DNA binding of the regulator DcuR of the fumarate-responsive two-component system DcuSR of Escherichia coli. Microbiology 150, 877-883.

Jensen, K. F. (1993). The Escherichia coli K-12 "wild types" W3110 and MG1655 have an rph frameshift mutation that leads to pyrimidine starvation due to low pyrE expression levels. J Bacteriol 175, 3401-3407.

Jensen, R. B., Wang, S. C. \& Shapiro, L. (2002). Dynamic localization of proteins and DNA during a bacterial cell cycle. Nat Rev Mol Cell Biol 3, 167-176.

Kaspar, S. \& Bott, M. (2002). The sensor kinase CitA (DpiB) of Escherichia coli functions as a high-affinity citrate receptor. Arch Microbiol 177, 313-321.

Kaspar, S., Perozzo, R., Reinelt, S., Meyer, M., Pfister, K., Scapozza, L. $\&$ Bott, M. (1999). The periplasmic domain of the histidine autokinase CitA functions as a highly specific citrate receptor. Mol Microbiol 33, 858-872.

Kentner, D., Thiem, S., Hildenbeutel, M. \& Sourjik, V. (2006). Determinants of chemoreceptor cluster formation in Escherichia coli. Mol Microbiol 61, 407-417.

Kneuper, H., Janausch, I. G., Vijayan, V., Zweckstetter, M., Bock, V., Griesinger, C. \& Unden, G. (2005). The nature of the stimulus and of the fumarate binding site of the fumarate sensor DcuS of Escherichia coli. J Biol Chem 280, 20596-20603.

Krämer, J., Fischer, J. D., Zientz, E., Vijayan, V., Griesinger, C., Lupas, A. \& Unden, G. (2007). Citrate sensing by the $\mathrm{C}_{4^{-}}$ dicarboxylate/citrate sensor kinase DcuS of Escherichia coli: binding site and conversion of DcuS to a $\mathrm{C}_{4}$-dicarboxylate- or citrate-specific sensor. J Bacteriol 189, 4290-4298.

Kulzer, F., Koberling, F., Christ, T., Mews, A. \& Basché, T. (1999). Terrylene in $p$-terphenyl: single-molecule experiments at room temperature. Chem Phys 247, 23-34.

Laemmli, U. K. (1970). Cleavage of structural proteins during the assembly of the head of bacteriophage T4. Nature 227, 680-685.

Liberman, L., Berg, H. C. \& Sourjik, V. (2004). Effect of chemoreceptor modification on assembly and activity of the receptor-kinase complex in Escherichia coli. J Bacteriol 186, 6643-6646.

Lybarger, S. R. \& Maddock, J. R. (2000). Differences in the polar clustering of the high- and low-abundance chemoreceptors of Escherichia coli. Proc Natl Acad Sci U S A 97, 8057-8062.

Maddock, J. R. \& Shapiro, L. (1993). Polar location of the chemoreceptor complex in the Escherichia coli cell. Science 259, 1717-1723.

Margolin, W. (2000). Green fluorescent protein as a reporter for macromolecular localization in bacterial cells. Methods 20, 62-72.

Mascher, T., Helmann, J. D. \& Unden, G. (2006). Stimulus perception in bacterial signal transducing histidine kinases. Microbiol Mol Biol Rev 70, 910-938.

Matsumoto, K., Kusaka, J., Nishibori, A. \& Hara, H. (2006). Lipid domains in bacterial membranes. Mol Microbiol 61, 1110-1117.

Miller, J. H. (1992). A Short Course in Bacterial Genetics. Cold Spring Harbor, NY: Cold Spring Harbor Laboratory.

Pappalardo, L., Janausch, I. G., Vijayan, V., Zientz, E., Junker, J., Peti, W., Zweckstetter, M., Unden, G. \& Griesinger, C. (2003). The NMR structure of the sensory domain of the membranous twocomponent fumarate sensor (histidine protein kinase) DcuS of Escherichia coli. J Biol Chem 278, 39185-39188.

Sambrook, J. \& Russell, D. W. (2001). Molecular Cloning: a Laboratory Manual, 3rd edn. Cold Spring Harbor, NY: Cold Spring Harbor Laboratory. 
Shapiro, L., McAdams, H. H. \& Losick, R. (2002). Generating and exploiting polarity in bacteria. Science 298, 1942-1946.

Silhavy, T. J., Berman, M. L. \& Enquist, L. W. (1984). Experiments with Gene Fusions. Cold Spring Harbor, NY: Cold Spring Harbor Laboratory.

Sourjik, V. (2004). Receptor clustering and signal processing in E. coli chemotaxis. Trends Microbiol 12, 569-576.

Sourjik, V. \& Berg, H. C. (2000). Localization of components of the chemotaxis machinery of Escherichia coli using fluorescent protein fusions. Mol Microbiol 37, 740-751.

Thiem, S., Kentner, D. \& Sourjik, V. (2007). Positioning of chemosensory clusters in E. coli and its relation to cell division. EMBO J 26, 1615-1623.

Towbin, H., Staehelin, T. \& Gordon, J. (1979). Electrophoretic transfer of proteins from polyacrylamide gels to nitrocellulose sheets: procedure and some applications. Proc Natl Acad Sci U S A 76, $4350-4354$.

Wheeler, R. T. \& Shapiro, L. (1999). Differential localization of two histidine kinases controlling bacterial cell differentiation. Mol Cell 4, 683-694.

Wingrove, J. A. \& Gober, J. W. (1996). Identification of an asymmetrically localized sensor histidine kinase responsible for temporally and spatially regulated transcription. Science 274, 597-601.

Yanisch-Perron, C., Vieira, J. \& Messing, J. (1985). Improved M13 phage cloning vectors and host strains: nucleotide sequences of the M13mp18 and pUC19 vectors. Gene 33, 103-119.

Zientz, E., Bongaerts, J. \& Unden, G. (1998). Fumarate regulation of gene expression in Escherichia coli by the DcuSR (dcuSR genes) twocomponent regulatory system. J Bacteriol 180, 5421-5425.

Edited by: W. Margolin 